\title{
Cytochrome P450 humanised mice
}

\author{
Frank J. Gonzalez \\ Laboratory of Metabolism, National Cancer Institute, Building 37, Room 3106B, Bethesda, MD 20892, USA \\ Correspondence to: Tel: +1 301496 9067; Fax: +1 301496 8419; E-mail: fjgonz@helix.nih.gov
}

Date received (in revised form): 26th March 2004

\begin{abstract}
Humans are exposed to countless foreign compounds, typically referred to as xenobiotics. These can include clinically used drugs, environmental pollutants, food additives, pesticides, herbicides and even natural plant compounds. Xenobiotics are metabolised primarily in the liver, but also in the gut and other organs, to derivatives that are more easily eliminated from the body. In some cases, however, a compound is converted to an electrophile that can cause cell toxicity and transformation leading to cancer. Among the most important xenobiotic-metabolising enzymes are the cytochromes P450 (P450s). These enzymes represent a superfamily of multiple forms that exhibit marked species differences in their expression and catalytic activities. To predict how humans will metabolise xenobiotics, including drugs, human liver extracts and recombinant P450s have been used. New humanised mouse models are being developed which will be of great value in the study of drug metabolism, pharmacokinetics and pharmacodynamics in vivo, and in carrying out human risk assessment of xenobiotics. Humanised mice expressing CYP2D6 and CYP3A4, two major drug-metabolising P450s, have revealed the feasibility of this approach.
\end{abstract}

Keywords: humanised mice, cytochromes P450, pharmacokinetics, bacterial artificial chromosome, transgenic mice

\section{Introduction}

Cytochromes P450 (P450s) and other foreign compound- or xenobiotic-metabolising enzymes are the primary interface between the chemical environment and the body. Xenobiotics include drugs, environmental contaminants and food additives. They can also encompass natural plant chemicals such as flavonoids, steroids and phytoalexins. Since there are countless different compounds that can enter an organism, there must be a means for their elimination. This is particularly important for hydrophobic chemicals, which can dissolve in lipids and accumulate in the body, or those that can react with cellular macromolecules and cause toxicity and cancer. In order to cope with the onslaught of chemical insults, mammals have evolved a large number of enzymes that appear to function in the metabolism of xenobiotics. Although some metabolism of endogenous chemicals has been found, the physiological relevance of these reactions is unknown. As a general paradigm, xenobiotic-metabolising enzymes convert lipid-soluble foreign chemicals into derivatives that can easily be eliminated from the body.

Xenobiotic-metabolising enzymes have historically been grouped into two functionally distinct classes, the phase 1 and phase 2 enzymes (Figure 1). Phase 1 enzymes include the P450s, flavin-containing monooxygenases (FMOs) and epoxide hydrolases $(\mathrm{EH})$. The phase 1 enzymes use $\mathrm{O}_{2}$ and reduced nicotinamide adenine dinucleotide phosphate in 'oxidation' reactions. The phase 2 enzymes, which include the glutathione S-transferases (GSTs), uridine diphosphate-glucuronosyltransferases (UGTs), N-acetyltransferases (NATs), sulfotransferases (SULTs), methyltransferases and a few others, add hydrophilic derivatives to compounds to make them more water soluble and more easily eliminated from the body. These conjugation reactions usually involve oxygen (hydroxyl or epoxide groups), nitrogen and sulphur atoms. Thus, xenobiotic-metabolising enzymes are responsible in large part for the disposal of foreign chemicals from the body. In the case of clinically used drugs, xenobiotic-metabolising enzymes generally metabolise and inactivate drugs; in the absence of metabolism, many drugs would stay in the body and remain biologically active for long periods of time, a property that would, in most cases, be unfavourable and lead to prolonged drug action. In some cases, prodrugs are converted to active metabolites by xenobiotic-metabolising enzymes. A list of xenobiotic-metabolising enzymes is shown in Table 1.

Paradoxically, the xenobiotic-metabolising enzymes are also responsible for converting inert chemicals to electrophilic derivatives that can cause cellular damage and transformation. For example, the P450s can produce electrophilic epoxides that react with cellular nucleophiles, including DNA. Phase 2 enzymes can also participate in activation reactions, in particular through the production of unstable esters derived from $\mathrm{N}$-oxides produced by P450s. ${ }^{8}$ Thus, some chemicals are 


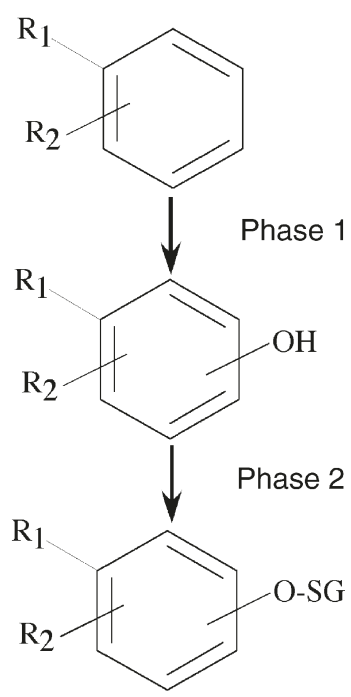

Figure I. Scheme depicting phase I and phase 2 drug metabolism. RI and R2 can represent any aliphatic or aromatic substituent. SG represents the results of a conjugation reaction by glutathione S-transferase.

inactivated while others are activated to electrophilic derivatives by xenobiotic metabolism.

The ability of xenobiotic-metabolising enzymes to handle many different types of chemicals is due to the fact that a single enzyme can metabolise a large number of different substrates. The substrate-binding site from a single P450 can accommodate different chemical structures. Oxidation can occur at different sites on the same molecule. There is also a high degree of overlapping substrate specificities among these enzymes, whereby a single compound can be metabolised by several enzymes. Thus, a limited number of P450s are able to metabolise scores of different chemicals, and this could account for the tremendous capacity for mammals with a limited number of enzymes to metabolise many different xenobiotics.

The xenobiotic-metabolising enzymes are important determinants for both the favourable (disease or symptom amelioration induced by drug action) and unfavourable (toxicity and carcinogenicity) responses to foreign chemicals. Patients can differ in both their responses to drugs and their susceptibility to chemically-induced toxicity due to the large degree of inter-individual differences in levels of expression. Since these enzymes activate carcinogens, there could be a difference in susceptibility to cancer which depends on levels of xenobiotic-metabolising enzymes. The latter has been examined by case control epidemiology studies that have attempted to determine the association between cancer and the presence of mutant alleles for xenobiotic-metabolising enzymes. 9,10

There are marked species differences in the expression and catalytic activities of xenobiotic-metabolising enzymes. As a result, rodents and humans can metabolise a single chemical entity quite differently. In fact, even rats and mice, which have been evolutionarily separated by at least 17 million years, have unique sets of xenobiotic-metabolising enzymes. In particular, the xenobiotic-metabolising $\mathrm{P} 450$ s show a high degree of species differences in the number of genes, regulation of genes and substrate specificities of individual enzymes. ${ }^{11}$ In humans, there are a limited number of $\mathrm{P} 450$ s that are known to metabolise clinically used drugs, and these enzymes exhibit substrate specificities and regulatory patterns that can markedly differ from P450s in mice and rats. In fact, cloning and sequencing of $\mathrm{P} 450$ s and, more recently, total genome

Table I. List of xenobiotic metabolising enzymes

\begin{tabular}{|c|c|}
\hline Enzymes & Reference \\
\hline \multicolumn{2}{|l|}{ Phase I } \\
\hline Cytochrome P450s (P450 or CYP) & http://drnelson.utmem.edu/CytochromeP450.html \\
\hline Flavin-containing monooxygenases (FMO) & I \\
\hline Epoxide hydrolases (mEH, sEH) & 2 \\
\hline \multicolumn{2}{|l|}{ Phase 2 'transferases' } \\
\hline Sulfotransferases (SULT) & 3 \\
\hline UDP-glucuronosyltransferases (UGT) & 4 \\
\hline Glutathione S-transferases (GST) & 5 \\
\hline NAD(P)H-quinone oxidoreductase (NQO) & 6 \\
\hline N-acetyltransferases (NAT) & 7 \\
\hline
\end{tabular}

$\mathrm{mEH}$ and $\mathrm{sEH}$ are microsomal and soluble epoxide hydrolase.

Abbreviations: UDP = uridine diphosphate; $\operatorname{NAD}(\mathrm{P}) \mathrm{H}=$ reduced nicotinamide adenine dinucleotide (phosphate). 
sequencing have revealed 102 putatively functional genes and 88 pseudogenes in the mouse and 57 putatively functional genes and 58 pseudogenes in the human. ${ }^{12}$ Thus, humans have fewer functional P450s genes than mice, an interesting fact that may help to develop a hypothesis on the driving forces of the evolution of xenobiotic-metabolising enzymes. ${ }^{11}$ The human P450s also exist in a number of allelic forms, and in some cases where there are mutant gene-inactivating mutations, these lead to P450 polymorphisms (http://www.imm.ki.se/CYPalleles). The major P450s involved in drug metabolism are CYP2C8, CYP2C9, CYP2C19, CYP2D6 and CYP3A4. Those P450s that are mainly involved in the metabolism of toxicants and carcinogens are CYP1A1, CYP1A2, CYP1B1, CYP2A6 and CYP2E1. The major polymorphic P450s are CYP2A6, CYP2C9, CYP2D6 and CYP2C19. Other P450s exhibit marked inter-individual differences in levels of expression which are not due to polymorphisms, notably CYP3A4. ${ }^{13}$

Since drugs are metabolised and inactivated by P450s, these enzymes dictate the rate of drug elimination. In many cases, a patient must take more than one medication, and this can result in drug interactions if both agents are metabolised by the same P450. This phenomenon, referred to as 'drug interactions', can lead to toxicity due to inhibition of metabolism and the resultant high and sustained serum levels of one of the drugs. Thus, it is critical to determine which P450 is responsible for metabolising a particular drug. In fact, it is now routine practice for pharmaceutical companies to establish which P450s metabolise a drug before it is subjected to clinical trials.

Over the past ten years, it has become standard practice to determine how a drug candidate is metabolised and which P450 form is responsible for its metabolism. This aids in the prediction of drug safety. In particular, by knowing how a drug is metabolised, it is possible to determine whether there will be drug interactions and whether there may be problems with inter-individual differences in metabolism and clearance caused by $\mathrm{P} 450$ polymorphisms. The best scenario for a drug company is to develop a drug that is metabolised by several forms of P450. To determine how a drug candidate is metabolised, human liver slices, human liver hepatocytes and human liver microsomes can be used in conjunction with inhibitors of specific P450 forms and antibodies to specific P450s. ${ }^{14}$ Recombinant human P450s, expressed in systems such as baculovirus, have also gained widespread use. ${ }^{15,16}$ In practice, both human liver-derived cells and extracts and recombinant $\mathrm{P} 450$ s are used to determine how a compound is metabolised. High-throughput robotic screening procedures have been developed using recombinant P450s in order to predict how drug candidates will be metabolised in humans. To determine which $\mathrm{P} 450$ has the highest rate of metabolism for a particular compound, standard Michaelis-Menton kinetics are used to estimate the relative rate of clearance or $\mathrm{Km} / \mathrm{Vmax}$. In addition, it should also be noted that computer modelling has been of some value in predicting metabolism with certain human P450s, such as CYP2C9 and CYP2D6. ${ }^{17}$

\section{Humanised mice}

Two strategies can be used to produce a humanised mouse. Historically, placing the cDNA behind a tissue-specific promoter has been used to make transgenic mice by standard pronuclei injection of recombinant DNA. For example, the rat albumin or mouse transthyretin promoter can be used to make transgenic mice that would allow for tissue-specific expression in liver. As an example, the human foetal CYP3A7 was expressed in liver using a metallothionein-1 promoter. $^{18}$ A second strategy for making humanised mice is to use genomic clones containing the complete gene upstream sequences that include all cis-acting regulatory elements. For example, a bacterial artificial chromosome (BAC) clone can be successfully used to generate a transgenic mouse. This would allow for tissue-specific and inducible regulation of the transgene and thus is the preferred method for making a humanised mouse that would be the most biologically predictive. The gene of choice can be found on the Celera or public human gene sequence databases, and a suitable BAC clone containing the full gene and its regulatory elements identified and used as a transgene. The BAC clone containing the full gene should not contain other gene open reading frames. Several transgenic founders (independent offspring derived from the egg injections) should be characterised to find a line that exhibits expression of the transgene in a tissue-specific manner that reflects the expression found in humans. In the experience of the author, $\mathrm{BAC}$ transgenes are stable and expressed over many generations.

\section{CYP2D6-humanised mice}

Mice contain nine Cyp2D genes; however, none of these genes appear to encode P450s with catalytic activities similar to CYP2D6, the only CYP2D gene product expressed in humans. ${ }^{12,19}$ In the absence of this activity, humanised mice can be developed by insertion of the CYP2D6 gene into the wild-type mouse genome. A CYP2D6-humanised mouse was produced using a lambda phage genomic clone containing the wild-type CYP2D6 gene. ${ }^{20}$ Interestingly, several founder lines were produced, all expressing CYP2D6 protein in liver. A couple of these lines also expressed the enzyme in kidney, however, and one expressed CYP2D6 in the liver, kidney and small intestine - known sites of expression of this P450 in humans. ${ }^{21}$ This latter line was used to determine the activity of CYP2D6 toward debrisoquine, an antihypertensive betaadrenoceptor blocking drug that is metabolised by CYP2D6 primarily through 4-hydroxylation. This reaction has been used for many years as an in vivo and in vitro metabolic probe for CYP2D6, since the discovery in 1977 of the CYP2D6 polymorphism. ${ }^{22}$ The humanised mice were able to efficiently metabolise debrisoquine, with pharmacokinetic parameters and urinary metabolite levels reflective of human extensive metabolisers of debrisoquine. ${ }^{21}$ To analyse the metabolism of debrisoquine in vivo, pharmacokinetic (PK) analysis was 
carried out. Mice are administered debrisoquine by oral gavage and blood samples taken at various times and subjected to analysis by liquid chromatography-mass spectrometry/mass spectrometry. These studies revealed that mice having two copies of the transgene cluster had a low area under the curve (AUC) for debrisoquine and a high AUC for 4hydroxydebrisoquine, while wild-type mice produced little of the metabolite and had a high AUC for the parent compound (Figure 2). Mice hemizygous for the transgene cluster were intermediate between wild-type and homozygote.

A second measure of debrisoquine metabolism in vivo is determination of the metabolic ratio (MR) of debrisoquine/ 4-hydroxydebrisoquine in the urine, the standard method for analysing debrisoquine metabolism in humans. This analysis revealed that the MR in wild-type mice was around 10 and that of CYP2D6-humanised mice, both homozygous and hemizygous, was about 1 ; the latter value is similar to that found in human extensive metabolisers. These studies confirm that wild-type mice are similar to human poor metabolisers of debrisoquine, and that CYP2D6-humanised mice are similar to extensive metabolisers. These studies establish the feasibility of using human genomic clones for the production of $\mathrm{P} 450$ humanised mice that exhibit in vivo metabolic activity that is similar to humans. Humanised mice have also been used to determine the mechanism of tissue-specific expression of CYP2D6. The CYP2D6 transgene was bred into a liver-specific null mouse for hepatocyte-enriched nuclear factor 4alpha (HNF4alpha). ${ }^{23}$ Mice lacking expression of HNF4alpha in the liver have markedly decreased expression of the CYP2D6 transgene, indicating a role for this factor in the control of the liver-specific expression of CYP2D6 (Figure 3). Expression is not lost in the absence of HNF4alpha, however, indicating that other liver-enriched factors may have a role in its expression.

CYP2D6 metabolises a large number of drugs that are used in the treatment of psychiatric disorders, including the antidepressants amitriptyline, fluoxetine and imipramine, and the neuroleptic agents haloperidol and risperidone. In addition, CYP2D6 carries out the demethylation of codeine to the more potent analgesic morphine. These data suggested that endogenous neurochemical substrates may exist for CYP2D6. Molecular modelling has been used to analyse CYP2D6 substrates. This revealed that 5-methoxytryptamine (5-MT) is a potential substrate for this P450. Indeed, 5-MT was found to be demethylated to 5-hydroxytryptamine (5-HT), also called serotonin, by recombinant CYP2D6 and transgenic CYP2D6. ${ }^{24}$ Conversion of 5-MT to 5-HT occurs
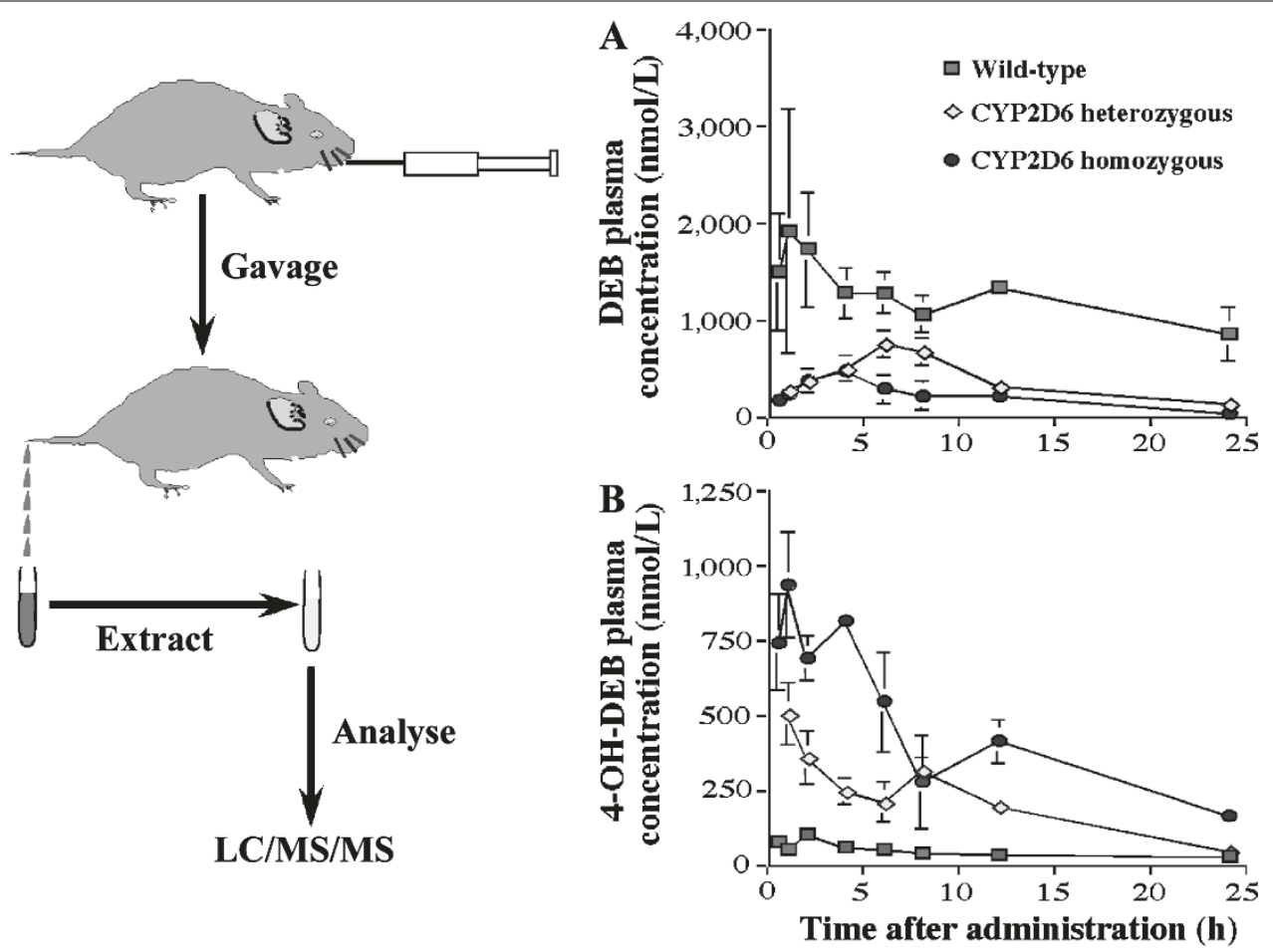

Figure 2. The pharmacokinetics of debrisoquine in CYP2D6-humanised mice. Time course of serum concentrations of (A) debrisoquine (DEB) and (B) 4-hydroxydebrisoquine (4-OH-DEB) from wild-type, CYP2D6-humanised heterozygous and CYP2D6-humanised homozygous mice after one single oral administration of DEB $(2.5 \mathrm{mg} / \mathrm{kg})$. Venous blood was obtained $0,0.5, \mathrm{I}, 2,4,6,8,12$ and 24 hours after DEB administration. Values represent the mean and the vertical lines the standard errors of the mean of DEB and 4-OHDEB from three mice. 


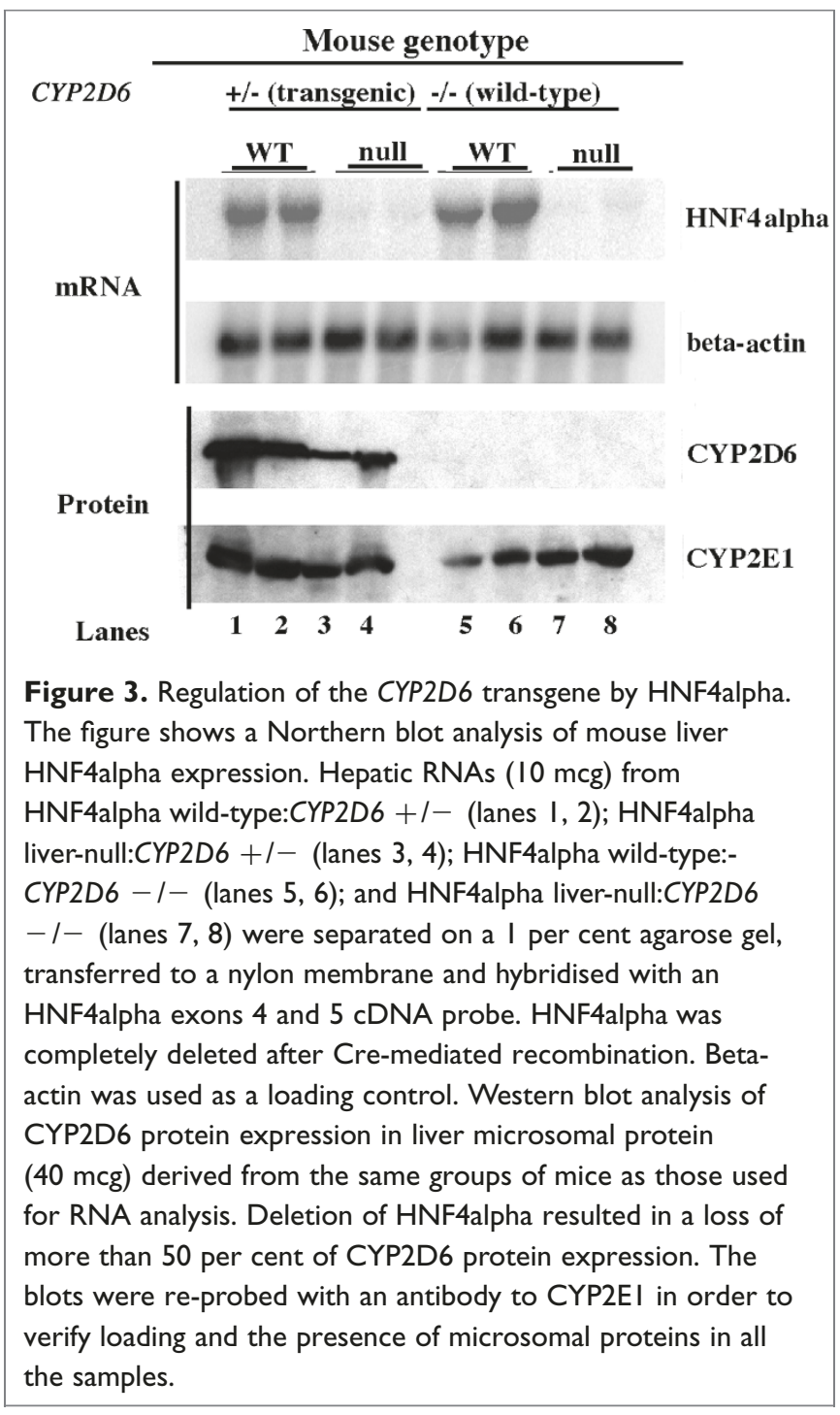

at a higher rate in the CYP2D6-humanised mice as compared with the wild-type mice. These studies revealed a new role for CYP2D6 in the serotonin-melatonin cycle.

\section{CYP3A4-humanised mice}

CYP3A4 is the most important of the human P450s for the metabolism of drugs. It is the most abundantly expressed P450 in human liver microsomes and is known to metabolise more than 60 per cent of all therapeutic drugs used in the treatment of many disorders, including hypercholesterolaemia (statin drugs), bacterial infections (erythromycin) and autoimmunity (cyclosporine). Thus, the potential for drug interactions is great and is of concern for the development of new drugs. ${ }^{25}$ In humans, four CYP3A P450s are expressed: CYP3A4, CYP3A5, CYP3A7 and CYP3A43. Interestingly, mice have eight $C Y P 3 A$ genes. ${ }^{12}$ The most abundantly expressed CYP3A gene in humans is CYP3A4. This $\mathrm{P} 450$ is especially of interest because it is not only expressed in liver, but also in the gut, where it can metabolise a large number of orally administered drugs.

To generate mice that express human CYP3A4, a BAC was used as a transgene. ${ }^{26}$ These mice expressed high levels of CYP3A4 protein in the small intestine. Surprisingly, little expression was found in the liver, a major site of CYP3A4 expression in humans; however, recent studies have revealed constitutive and inducible expression in the livers of female mice (Yu et al., unpublished results). To evaluate CYP3A4 catalytic activity, the drug midazolam was used, since it is a standard probe for CYP3A4 activity in humans. ${ }^{27}$ Midazolam is oxidised at the 4 and $1^{\prime}$ positions by CYP3A4. The pharmacokinetics of midazolam metabolism were determined upon oral administration of the drug. Differences in AUC for the parental compound and the primary $1^{\prime}$-hydroxymidazolam were detected between the CYP3A4-humanised and wildtype mice, indicating that the trangenics have a higher rate of midazolam metabolism and clearance (Figure 4). No differences were observed between the transgenic mice and wildtype mice when the drug was administered intravenously. These mice will aid in the in vivo analysis of orally administered drugs that are substrates for CYP3A4. They will also be of value in determining the mechanism of tissue-specific and inducible regulation of the CYP3A4 gene.

\section{Conclusions}

Xenobiotic-metabolising enzymes are the interface between humans and their chemical environment. While they are absolutely required for the elimination of clinically used drugs, they can lead to adverse drug effects due to drug interactions and polymorphisms. These enzymes are also responsible for the activity of chemical carcinogens. Thus, drugs and chemicals to be used in humans must carefully be tested to determine how they will be metabolised. While in vitro systems have been developed to predict human metabolism of drugs, analysis of drug metabolism in vivo, using rodents, can lead to uncertainty due to species differences in xenobiotic-metabolising enzymes. Humanised mice expressing human P450s can be used to fill the gap between in vitro and in vivo testing systems. The CYP2D6and CYP3A4-humanised mouse models validate the production and use of these new models for the study of in vivo pharmacogenetics. The challenge remains, however, to produce fully humanised mice in which the endogenous mouse genes are knocked out. While targeted gene disruption has been used to knock out the CYP1 family and CYP2E1, most of the CYP2 subfamilies in mice consist of multiple genes. For example, the $C Y P 2$ and CYP3A loci in mice consist of nine and eight genes, respectively, spanning hundreds of kilobases of DNA. Thus, standard gene knockout technology cannot be used to delete the whole subfamily. While each gene can be disrupted, 

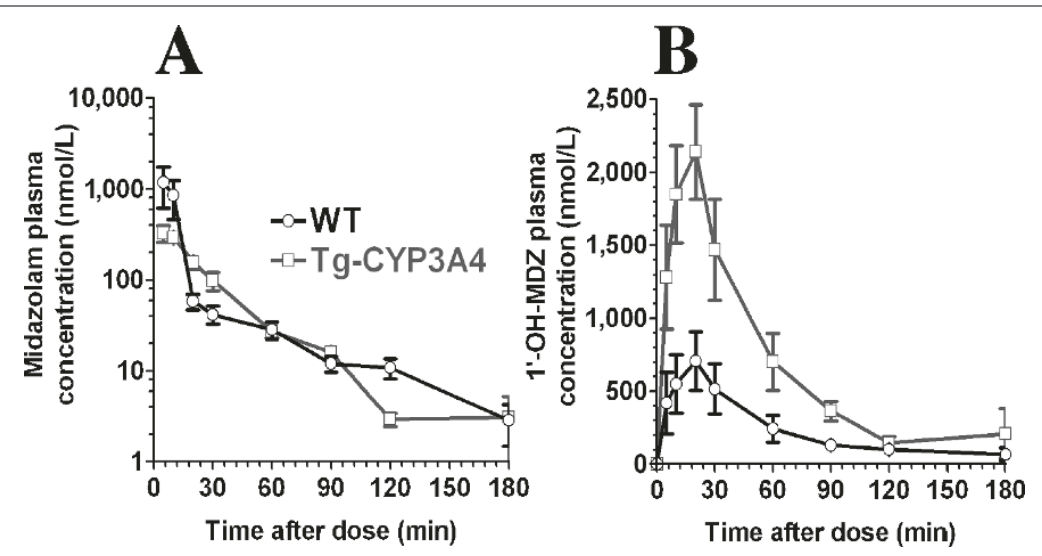

Figure 4. The pharmacokinetics of midazolam in CYP3A4-humanised mice. The graphs show the time course of the serum concentrations of (A) midazolam and (B) I'-hydroxymidazolam (I'-OH-MDZ) from wild-type (WT), CYP3A4-humanised mice (Tg-CYP3A4) after one single oral administration of midazolam. Venous blood was obtained 0, 0.5, I, 2, 4, 6, 8, 12 and 24 hours after administration. Values represent the mean and the vertical lines the standard errors of the mean of midazolam and I'-OH-MDZ from three mice. Note the higher maximal concentration (Cmax) for midazolam in WT mice compared with Tg-CYP3A4 mice.

the modified genes can never be joined on a single chromosome due to their proximities. Nevertheless, the Cre-loxP strategy is a method that can allow a large segment of DNA containing the whole $\mathrm{P} 450$ subfamily to be deleted. ${ }^{28}$ LoxP sites are inserted at each end of the P450 locus by consecutive transfection of

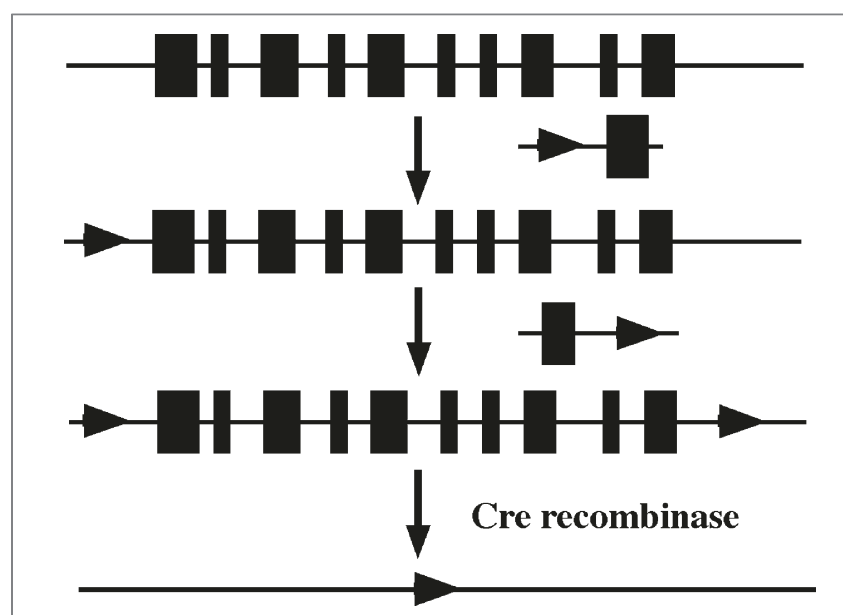

Figure 5. Use of the Cre-loxP strategy to delete an entire subfamily of P450 genes. The individual genes are denoted by rectangles and the loxP sites by triangles. The sequence of the locus can be obtained from the public or Celera database, and bacterial artificial chromosome clones that flank the complete cluster of genes can be chosen. LoxP sites are introduced by use of in vivo recombination to generate constructs for transfection into embryonic stem (ES) cells. ${ }^{29}$ This is carried out by two consecutive transfections into ES cells followed by treatment with Cre recombinase to delete the DNA between the loxP sites. The ES cells can then be used to make a knockout mouse. recombinant DNAs into embryonic stem (ES) cells using standard protocols (Figure 5). ${ }^{29}$ The intervening segment of DNA can be deleted in ES cells using transfected Cre recombinase.

Alternatively, deletion can be carried out in the mouse embryo or adult mouse using a Cre recombinase transgene. By use of this method, all of the non-essential xenobiotic-metabolising P450s can be deleted and replaced with their human counterparts, leading to a fully P450-humanised mouse.

\section{(C) United States Federal Government}

\section{References}

1. Ziegler, D.M. (2002), 'An overview of the mechanism, substrate specificities, and structure of FMOs', Drug Metab. Rev. Vol. 34, pp. 503-511.

2. Fretland, A.J. and Omiecinski, C.J. (2000), 'Epoxide hydrolases: Biochemistry and molecular biology', Chem. Biol. Interact. Vol. 129, pp. 41-59.

3. Nagata, K. and Yamazoe, Y. (2000), 'Pharmacogenetics of sulfotransferase', Annu. Rev. Pharmacol. Toxicol. Vol. 40, pp. 159-176.

4. King, C.D., Rios, G.R., Green, M.D. et al. (2000), 'UDP-glucuronosyltransferases', Curr. Drug Metab. Vol. 1, pp. 143-161.

5. Strange, R.C., Spiteri, M.A. and Ramachandran, S. (2001), 'GlutathioneS-transferase family of enzymes', Mutat. Res. Vol. 482, pp. 21-26.

6. Ross, D., Kepa, J.K. and Winski, S.L. (2000), 'NAD(P)H:quinone oxidoreductase 1 (NQO1): Chemoprotection, bioactivation, gene regulation and genetic polymorphisms', Chem. Biol. Interact. Vol. 129, pp. 77-97.

7. Hein, D.W. (2002), 'Molecular genetics and function of NAT1 and NAT2: Role in aromatic amine metabolism and carcinogenesis', Mutat. Res. Vol. 506/507, pp. 65-77.

8. Guengerich, F.P. and Shimada, T. (1998), 'Activation of procarcinogens by human cytochrome P450 enzymes', Mutat. Res. Vol. 400, pp. 201-213.

9. Sheweita, S.A. and Tilmisany, A.K. (2003), 'Cancer and phase II drugmetabolizing enzymes', Curr. Drug Metab. Vol. 4, pp. 45-58.

10. Clapper, M.L. (2000), 'Genetic polymorphism and cancer risk', Curr. Oncol. Rep. Vol. 2, pp. 251-256.

11. Gonzalez, F.J. and Nebert, D.W. (1990), 'Evolution of the P450 gene superfamily: Animal-plant warfare, molecular drive and human genetic differences in drug oxidation', Trends Genet. Vol. 6, pp. 182-186. 
12. Nelson, D.R., Zeldin, D.C., Hoffman, S.M.G. et al. (2004), 'Comparison of cytochrome P450 (CYP) genes from the mouse and human genomes, including nomenclature recommendations for genes, pseudogenes and alternative-splice variants', Pharmacogenetics Vol. 14, pp. 1-18.

13. Lamba, J.K., Lin, Y.S., Schuetz, E.G. et al. (2002), 'Genetic contribution to variable human CYP3A-mediated metabolism', Adv. Drug Deliv. Rev. Vol. 54, pp. 1271-1294.

14. Parkinson, A. (1996), 'An overview of current cytochrome P450 technology for assessing the safety and efficacy of new materials', Toxicol. Pathol. Vol. 24, pp. $48-57$.

15. Gonzalez, F.J. and Korzekwa, K.R. (1995), 'Cytochromes P450 expression systems', Annu. Rev. Pharmacol. Toxicol. Vol. 35, pp. 369-390.

16. Crespi, C.L. and Miller, V.P. (1999), 'The use of heterologously expressed drug metabolizing enzymes - State of the art and prospects for the future', Pharmacol. Ther. Vol. 84, pp. 121-131.

17. Lewis, D.F. (2003), 'P450 structures and oxidative metabolism of xenobiotics', Pharmacogenomics Vol. 4, pp. 387-395.

18. Li, Y., Yokoi, T., Kitamura, R. et al. (1996), 'Establishment of transgenic mice carrying human fetus-specific CYP3A7', Arch. Biochem. Biophys. Vol. 329 , pp. $235-240$.

19. Masubuchi, Y., Iwasa, T., Hosokawa, S. et al. (1997), 'Selective deficiency of debrisoquine 4-hydroxylase activity in mouse liver microsomes', J. Pharmacol. Exp. Ther. Vol. 82, pp. 1435-1441.

20. Kimura, S., Umeno, M., Skoda, R.C. et al. (1989), 'The human debrisoquine 4-hydroxylase (CYP2D) locus: Sequence and identification of the polymorphic CYP2D6 gene, a related gene, and a pseudogene', Am. J. Hum. Genet. Vol. 45, pp. 889-904.
21. Corchero, J., Granvil, C.P., Akiyama, T.E. et al. (2001), 'The CYP2D6 humanized mouse: Effect of the human CYP2D6 transgene and HNF4alpha on the disposition of debrisoquine in the mouse', Mol. Pharmacol. Vol. 60, pp. 1260-1267.

22. Mahgoub, A., Idle, J.R. and Dring, L.G. (1977), 'Polymorphic hydroxylation of debrisoquine in man', Lancet Vol. 2, pp. 584-586.

23. Hayhurst, G.P., Lee, Y.H., Lambert, G. et al. (2001), 'Hepatocyte nuclear factor 4alpha (nuclear receptor 2A1) is essential for maintenance of hepatic gene expression and lipid homeostasis', Mol. Cell Biol. Vol. 21, pp. 1393-1403.

24. Yu, A.M., Idle, J.R., Byrd, L.G. et al. (2003), 'Regeneration of serotonin from 5-methoxytryptamine by polymorphic human CYP2D6', Pharmacogenetics Vol. 13, pp. 173-181.

25. Rendic, S. (2002), 'Summary of information on human CYP enzymes: Human P450 metabolism data', Drug Metab. Rev. Vol. 34, pp. 83-448.

26. Granvil, C.P., Yu, A.M., Elizondo, G. et al. (2003), 'Expression of the human CYP3A4 gene in the small intestine of transgenic mice: In vitro metabolism and pharmacokinetics of midazolam', Drug Metab. Dispos. Vol. 31, pp. $548-558$.

27. Thummel, K.E., Shen, D.D., Podoll, T.D. et al. (1994), 'Use of midazolam as a human cytochrome P450 3A probe: I. In vitro-in vivo correlations in liver transplant patients', J. Pharmacol. Exp. Ther. Vol. 271, pp. 549-556.

28. Kuhn, R. and Torres, R.M. (2002), 'Cre/loxP recombination system and gene targeting', Methods Mol. Biol. Vol. 180, pp. 175-204.

29. Liu, P., Jenkins, N.A. and Copeland, N.G. (2002), 'Efficient Cre-loxPinduced mitotic recombination in mouse embryonic stem cells', Nat. Genet. Vol. 30, pp. 66-72. 